\title{
TRACKING DEPRESSION IN ANKYLOSING SPONDYLITIS USING PHQ-9 QUESTIONNAIRE
}

Adah Sophia Rodrigues Vieira ${ }^{1 *}$, Guilherme Lopes Pereira ${ }^{1}$, João Guilherme Farias de Sá Gomes ${ }^{1}$, Amanda Alexia Rodrigues Vieira ${ }^{1}$, Pedro Ferreira Ferro Neto ${ }^{2}$, Rejane Maria Rodrigues de Abreu Vieira ${ }^{1,3}$

1.Universidade de Fortaleza, Fortaleza (CE), Brazil. 2.Centro Universitário Christus, Fortaleza (CE), Brazil. 3.Hospital Geral de Fortaleza, Fortaleza (CE), Brazil.

${ }^{\star}$ Corresponding author: adahsophia0820@gmail.com

\section{BACKGROUND}

Ankylosing spondylitis (AS) is a chronic disease that tackles young age adults, with important implications on the patient's physical and mental health. Chronic pain and deformities resulting from this diagnosis tend to show many associations with a depressive framework. The use of instruments to evaluate possible depression diagnosis on these patients, with proper and specific orientation, is of great importance. The Patient Health Questionnaire-9 (PHQ-9) is a questionnaire that foresees the presence of each one of the symptoms that characterize a major depressive disorder, described at the Diagnostic and Statistical Manual of Mental Disorders (DSM-V).

\section{MATERIALS AND METHODS}

Transversal and quantitative study, conducted at the rheumatology ambulatory of a public hospital. Ankylosing spondylitis patients, according to the modified New York criteria, were available using the PHQ-9 questionnaire. The ethics committee approved, and all participants signed the free and clarified consent term.

\section{RESULTS}

The study included a total of 104 patients, in which $74 \%$ were male. Sixty percent were below 45 years, and $68 \%$ had the diagnosis for more than six years. Ten percent were smokers, and alcohol usage was observed on $21 \%$ of the sample. Thirty percent were unemployed, $27 \%$ retired, and $43 \%$ were professionally active. A total of 38 patients (36.5\%) scored above 9 on the PHQ-9, indicative of a problematic depressive state, and 6 scored above 20, which is considered criteria for significant depression disorder. The factors considered to be associated with a depressive state were an extended disease and higher schooling levels. Alcohol usage, smoking, and exercise did not show any association with the depressive state.

\section{CONCLUSION}

Ankylosing spondylitis, with its chronic and disabling nature, shows high relation with depression. The PHQ-9 is a simple questionnaire to apply that can benefit from tracking signs and symptoms of this pathology, allowing a more proper conduct for these patients, while preserving their quality of life. 